\title{
Renormalized energy of ground and first excited state of Fröhlich polaron in the range of weak coupling
}

\author{
M.V. Tkach|; Ju.O. Seti, O.M. Voitsekhivska, O.Yu. Pytiuk \\ Chernivtsi National University, 2 Kotsyubinsky St., 58012 Chernivtsi, Ukraine
}

Received June 10, 2015, in final form July 2, 2015

Partial summing of infinite range of diagrams for the two-phonon mass operator of polaron described by Fröhlich Hamiltonian is performed using the Feynman-Pines diagram technique. Renormalized spectral parameters of ground and first excited (phonon repeat) polaron state are accurately calculated for a weak electron-phonon coupling at $T=0 \mathrm{~K}$. It is shown that the stronger electron-phonon interaction shifts the energy of both states into low-energy region of the spectra. The ground state stays stationary and the excited one decays at a bigger coupling constant.

Key words: polaron, phonon, electron-phonon interaction, Green's function, mass operator

PACS: $71.38 .-k, 63.20 . k d, 63.20 . d k, 72.10 . D i$

\section{Introduction}

The concept of polaron, as electron interacting with the polarization vibrations of the crystal, introduced by Landau in 1933 [1 2] has attracted a permanent attention lately. For a long time, the theory of polaronic phenomena was developed in the framework of different physical models using various mathematical approaches [3 4]. Following the Fröhlich's introduction of the Hamiltonian of electron interacting with nondispersive (optical) phonons of a dielectric medium via its polarization, in representation of second quantization [5], the methods of quantum field theory were used to solve the polaron problems [6-8]. Studying the renormalized energy of ground state and the effective mass of structureless Fröhlich polaron, three ranges for electron-phonon coupling were established. The mobility, impedance and optical conductivity were investigated in detail for these ranges.

Almost fifty years ago J. Devreese with colleagues [9, 10] were investigating the polaron complexes related to the excited states of electron-phonon system. In particular, it was established that in the regimes of intermediate and strong coupling, the so-called relaxed excited states (RES) exist [9-13] in the region of energies a little bit bigger than the renormalized energy of the ground polaron state plus the energy of one phonon while the Franck-Condon (FC) excited states are located higher in the energy scale. The treatment of the structure of the excited quasi-stationary states spectra is a complicated problem, constantly attracting attention of theoretical community. The results obtained within different methods were compared in original papers [14-16] and in reviews [17-19].

In the process of theoretical investigation of electron-phonon systems, the physical picture was studied in detail and a new mathematical approach [14-16] made it possible to avoid the contradictive results previously obtained in the approximations used in earlier papers. The exact diagrammatic quantum Monte Carlo (DMC) method [14] was used in order to solve the problem of Fröhlich polaron RES and FC states and analyze their dependence on the regime of coupling. In the cited papers it was proven that the one-phonon approximation, used in the early papers of J. Devreese with colleagues, was not capable of correctly describing the optical conductivity in the limit of strong coupling because, in particular, the

\footnotetext{
*E-mail: ktf@chnu.edu.ua
} 
energy density for RES and FC states was not correctly defined even at a very small coupling constant $(\alpha=0.05)$. Further, in reference [16], the main results of reference [14] were confirmed by DMC method for a wide range of the coupling constant. These results correlated well with the ones obtained in the memory function formalism (MFF) and strong coupling expansion (SCE) which assumed the FC principle.

We should mention that DMC method [14 17] made it possible to establish the properties of optical conductivity in the region of excited states of Fröhlich polaron and was used to study the hightemperature superconductivity. In particular, in reference [20] it was proven that electron-phonon interaction, together with magnetic sub-system plays a substantial role in the formation of high-temperature superconductivity.

The search for mathematical approaches to the study of the excited states of Fröhlich polarons with intermediate and strong coupling overshadows the solution of the same problems in the range of weak electron-phonon coupling for 3D systems. However, with the appearance of low dimensional nano-systems (quantum dots, quantum wires and quantum layers, characterized by weak coupling), where the difference between electron energies resonates with the energy of confined optical and interface phonons, the attention payed to the excited polaron states has grown essentially [18, 21-27]. Now it is necessary to study the renormalized spectrum of excited states of 3D polaron in the range of weak coupling.

From the papers [5, 8,22$]$ we know that at $\alpha \ll 1$ in one-phonon approximation for the mass operator, the energy of polaron ground state $(E)$ at $T=0 \mathrm{~K}$ shifts into the low-energy region due to the electronphonon interaction. In the vicinity of $E+\Omega$ energies, where $\Omega$ is the polarization phonon energy, there is observed a wide peak, related to the bound state of polaron with one phonon. This fact is clear and coordinates with physical considerations. However, when the coupling constant increases, the energy of excited state shifts into the opposite side of the spectrum, contradicting physical consideration because at $T=0 \mathrm{~K}$ the virtual phonons are not capable of providing their energy to create a new quasi-particle. So, it is clear that one-phonon approximation is not valid for an accurate calculation of mass operator (MO). Thus, the further approximation was to take into account the two two-phonon diagrams, proportional to $\alpha^{2}$ in the polaron Green's function, besides the one-phonon, proportional to $\alpha$. Herein, the magnitude of renormalized energy of the ground state became more accurate (the red shift increased) but the peak of the energy in the region of the bound state, being located in the left-hand part respectively to that in one-phonon approximation, shifted into the high-energy region when $\alpha$ increased.

The abovementioned result brings us to the conclusion that the finite number of diagrams being taken into account in polaron MO is insufficient to obtain the correct physical behavior of the excited polaron state when the coupling constant varies in a wide range. It is evident that one has to perform a partial summing of infinite number of diagrams of the respective order.

In this paper we study the renormalized energies of Fröhlich polaron with weak electron-phonon coupling in such approximation for the MO, which correctly takes into account partially summed one- and two-phonon diagrams over all orders of the coupling constant. The result of this approach is that using the Feynman-Pines diagram technique we obtained a physically correct conclusion: both the ground and the first excited polaron states for a system with weak coupling shift into the low-energy region when $\alpha$ increases.

\section{The Fröhlich polaron at $\boldsymbol{T}=\mathbf{0} \mathrm{K}$}

We consider polaron as an electron interacting with polarization phonons described by Fröhlich Hamiltonian

$$
H=\sum_{\vec{k}} E_{\vec{k}} a_{\vec{k}}^{+} a_{\vec{k}}+\sum_{\vec{q}} \Omega_{\vec{q}}\left(b_{\vec{q}}^{+} b_{\vec{q}}+\frac{1}{2}\right)+\sum_{\vec{k}, \vec{q}} \varphi(\vec{q}) a_{\vec{k}+\vec{q}}^{+} a_{\vec{k}}\left(b_{\vec{q}}+b_{-\vec{q}}^{+}\right),
$$

where

$$
E_{\vec{k}}=E+\frac{\hbar^{2} k^{2}}{2 m}, \quad \Omega_{\vec{q}}=\Omega
$$

are the energies of electron and optical phonons, respectively,

$$
\varphi(q)=\frac{\mathrm{i}}{q} \sqrt{\frac{2 \pi \hbar \Omega \alpha}{V} \sqrt{\frac{2 \Omega}{m}}}
$$


— their binding function expressed within the coupling constant

$$
\alpha=\frac{e^{2}}{\hbar}\left(\frac{1}{\varepsilon_{\infty}}-\frac{1}{\varepsilon_{0}}\right) \sqrt{\frac{m}{2 \Omega}}=\left(\frac{1}{\varepsilon_{\infty}}-\frac{1}{\varepsilon_{0}}\right) \sqrt{\frac{m}{m_{0}} \frac{\mathrm{Ry}}{\Omega}},
$$

with $m_{0}$ - the mass of electron in vacuum and Ry $=13.6 \mathrm{eV}$.

It is well known [28] that at $T=0 \mathrm{~K}$, the renormalized electron-phonon spectrum is obtained from the poles of Fourier image of polaron Green's function which, in its turn, through the Dyson equation

$$
G(\vec{k}, \omega)=\left\{\hbar \omega-E_{\vec{k}}-M(\vec{k}, \omega)\right\}^{-1}
$$

is related with MO expressed in a diagrammatic form. An analytical calculation of MO in two first orders over the powers of the coupling constant $\alpha$ is performed. According to the rules of Feynman-Pines diagram technique, MO of the first order over $\alpha$ is defined as

$$
\underset{\vec{k}-\vec{q}}{\stackrel{\vec{q}}{\longrightarrow}}=M_{1}(\vec{k}, \omega)=\sum_{\vec{q}} \frac{|\varphi(\vec{q})|^{2}}{\hbar \omega-E_{\vec{k}-\vec{q}}-\Omega+\mathrm{i} \eta} .
$$

Transiting from summing to integration over $\vec{q}$ and accounting for (2) and (3), we obtain

$$
M_{1}(\vec{k}, \omega)=\frac{e^{2} \Omega}{4 \pi^{2}}\left(\frac{1}{\varepsilon_{\infty}}-\frac{1}{\varepsilon_{0}}\right) \int \frac{\mathrm{d}^{3} \vec{q}}{q^{2}\left[\hbar \omega-E-\frac{\hbar^{2}}{2 m}(\vec{k}-\vec{q})^{2}-\Omega+\mathrm{i} \eta\right]} .
$$

Further, it is convenient to introduce the dimensionless MO $m=M / \Omega$, with dimensionless energy $\xi$ and quasi-momentum $(\vec{K}$ and $\vec{Q})$

$$
\xi=\frac{\hbar \omega-E}{\Omega}, \quad \vec{K}=\frac{\hbar}{\sqrt{2 m \Omega}} \vec{k}, \quad \vec{Q}=\frac{\hbar}{\sqrt{2 m \Omega}} \vec{q} .
$$

In these variables the expression (7) is rewritten as follows:

$$
m_{1}(\vec{K}, \xi)=\frac{\alpha}{2 \pi^{2}} \int_{-\infty}^{\infty} \frac{\mathrm{d}^{3} \vec{Q}}{Q^{2}\left[\xi-1-(\vec{K}-\vec{Q})^{2}+\mathrm{i} \eta\right]}
$$

Integrating in the spherical coordinate system, an exact analytical expression is obtained

$$
m_{1}(K, \xi)=-\frac{\alpha}{K} \begin{cases}\arctan \left(\frac{K}{\sqrt{1-\xi}}\right), & \xi \leqslant 1, \\ \frac{\mathrm{i}}{2} \ln \left|\frac{\sqrt{\xi-1}+K}{\sqrt{\xi-1}-K}\right|+\frac{\pi}{2} \theta(K-\sqrt{\xi-1}), & \xi \geqslant 1 .\end{cases}
$$

Herein, at $K=0$

$$
\operatorname{Re} m_{1}(\xi)=-\frac{\alpha \theta(1-\xi)}{\sqrt{1-\xi}}, \quad \operatorname{Im}_{1}(\xi)=-\frac{\alpha \theta(\xi-1)}{\sqrt{\xi-1}} .
$$

The analysis of MO $m_{1}(K, \xi)$ and of the peculiarities of polaron spectra will be performed further on. Now we should note that as far as in $m_{1}(K, \xi)$ the electron interacts only with one virtual phonon, it is called a one-phonon MO. Diagram technique proves that contrary to the two-, three- and $n$-phonon MO, $m_{1}(K, \xi)$ is a unitary one, which does not contain an infinite number of terms. The other terms of complete MO contain an infinite number of diagrams over all powers of coupling constant. 
Now we observe the MO of the second order over the power of the coupling constant, corresponding to the diagram without an intersection of phonon lines. According to the rules of diagram technique, it is written as follows:

$$
M_{2}^{a}(\vec{k}, \omega)=\underset{\vec{k}-\vec{q}_{1} \vec{k}-\vec{q}_{1}-\vec{q}_{2} \vec{k}-\vec{q}_{1}}{\stackrel{\vec{q}_{1}, \vec{q}_{2}}{\longrightarrow}}=\sum_{\vec{q}_{1}} \frac{\varphi^{2}\left(\vec{q}_{1}\right) \varphi^{2}\left(\vec{q}_{2}\right)}{\left(\hbar \omega-E_{\vec{k}-\vec{q}_{1}}-\Omega+\mathrm{i} \eta\right)^{2}\left(\hbar \omega-E_{\vec{k}-\vec{q}_{1}-\vec{q}_{2}}-2 \Omega+\mathrm{i} \eta\right)} .
$$

Transiting here from summing to integration, accounting for (2) and (3) and using the dimensionless parameters, we obtain

$$
m_{2}^{a}(\vec{K}, \xi)=\frac{\alpha^{2}}{4 \pi^{4}} \int_{-\infty}^{\infty} \frac{\mathrm{d}^{3} \vec{Q}_{1}}{Q_{1}^{2}\left[\xi-1-\left(\vec{K}-\vec{Q}_{1}\right)^{2}+\mathrm{i} \eta\right]^{2}} \int_{-\infty}^{\infty} \frac{\mathrm{d}^{3} \vec{Q}_{2}}{Q_{2}^{2}\left[\xi-2-\left(\vec{K}-\vec{Q}_{1}-\vec{Q}_{2}\right)^{2}+\mathrm{i} \eta\right]}
$$

We are going to study the renormalized energies of the bottom of the ground and excited states of polaron. Thus, here and further we put $\vec{K}=0$ in order to simplify the analytical calculations. As a result, the integration in 13 is performed exactly and in the region $\xi \leqslant 1$ the expression for $m_{2}^{a}(\xi \leqslant 1)$ containing only the real part is obtained in the following form:

$$
m_{2}^{a}(\xi \leqslant 1)=-\frac{\alpha^{2}}{(1-\xi)^{2}}\left[\ln \left(1+\sqrt{\frac{1-\xi}{2-\xi}}\right)-\frac{\sqrt{1-\xi}}{2(\sqrt{1-\xi}+\sqrt{2-\xi})}\right] .
$$

Continuing analytically this expression into the region $1 \leqslant \xi \leqslant 2$, one can obtain both the real and the imaginary parts, while in the region $\xi \geqslant 2$, only the real part [22].

The MO of the second order over the power of the coupling constant, corresponding to the diagram with the intersection of phonon lines has the following form:

$$
\begin{aligned}
M_{2}^{b}(\vec{k}, \omega) & =\underset{\vec{k}-\vec{q}_{1} \vec{k}-\vec{q}_{1}-\vec{q}_{2} \vec{k}-\vec{q}_{2}}{\stackrel{\vec{q}_{1}}{\vec{q}_{2}}} \\
& =\sum_{\vec{q}_{1}, \vec{q}_{2}} \frac{\varphi^{2}\left(\vec{q}_{1}\right) \varphi^{2}\left(\vec{q}_{2}\right)}{\left(\hbar \omega-E_{\vec{k}-\vec{q}_{1}}-\Omega+i \eta\right)^{2}\left(\hbar \omega-E_{\vec{k}-\vec{q}_{1}-\vec{q}_{2}}-2 \Omega+i \eta\right)\left(\hbar \omega-E_{\vec{k}-\vec{q}_{2}}-\Omega+i \eta\right)} .
\end{aligned}
$$

In dimensionless variables it is rewritten as follows:

$$
\begin{aligned}
m_{2}^{b}(\vec{K}, \xi)= & \frac{\alpha^{2}}{4 \pi^{4}} \int_{-\infty}^{\infty} \frac{\mathrm{d}^{3} \vec{Q}_{1}}{Q_{1}^{2}\left[\xi-1-\left(\vec{K}-\vec{Q}_{1}\right)^{2}+\mathrm{i} \eta\right]} \\
& \times \int_{-\infty}^{\infty} \frac{\mathrm{d}^{3} \vec{Q}_{2}}{Q_{2}^{2}\left[\xi-1-\left(\vec{K}-\vec{Q}_{2}\right)^{2}+\mathrm{i} \eta\right]\left[\xi-2-\left(\vec{K}-\vec{Q}_{1}-\vec{Q}_{2}\right)^{2}+\mathrm{i} \eta\right]}
\end{aligned}
$$

At $\vec{K}=0$, integration in 16 is performed exactly. As a result, in the region $\xi \leqslant 1$ the expression for $m_{2}^{b}(\xi \leqslant 1)$ containing only the real part is obtained

$$
m_{2}^{b}(\xi \leqslant 1)=-\frac{\alpha^{2}}{(1-\xi)^{2}} \ln \left[\frac{\sqrt{2-\xi}+\sqrt{1-\xi}}{\xi \sqrt{2-\xi}+(2-\xi) \sqrt{1-\xi}}\right]
$$

Continuing analytically this expression into the region $1 \leqslant \xi \leqslant 2$, one can obtain both the real and the imaginary parts while in the region $\xi \geqslant 2$, only the real part [22]. 
Finally, the functions $m_{2}^{a}(\xi \leqslant 1)$ and $m_{2}^{b}(\xi \leqslant 1)$ and their analytical continuations completely define the MO of the second order over the power of the coupling constant

$$
\bar{m}_{2}^{(2)}(\xi)=m_{2}^{a}(\xi)+m_{2}^{b}(\xi)
$$

as a complex function of dimensionless energy $\xi$ in the whole range of its variation.

The analytical expressions prove that $m_{1}(\xi)$ has a discontinuity at $\xi=1$ while $\bar{m}_{2}^{(2)}(\xi)-$ at $\xi=1$ and $\xi=2$.

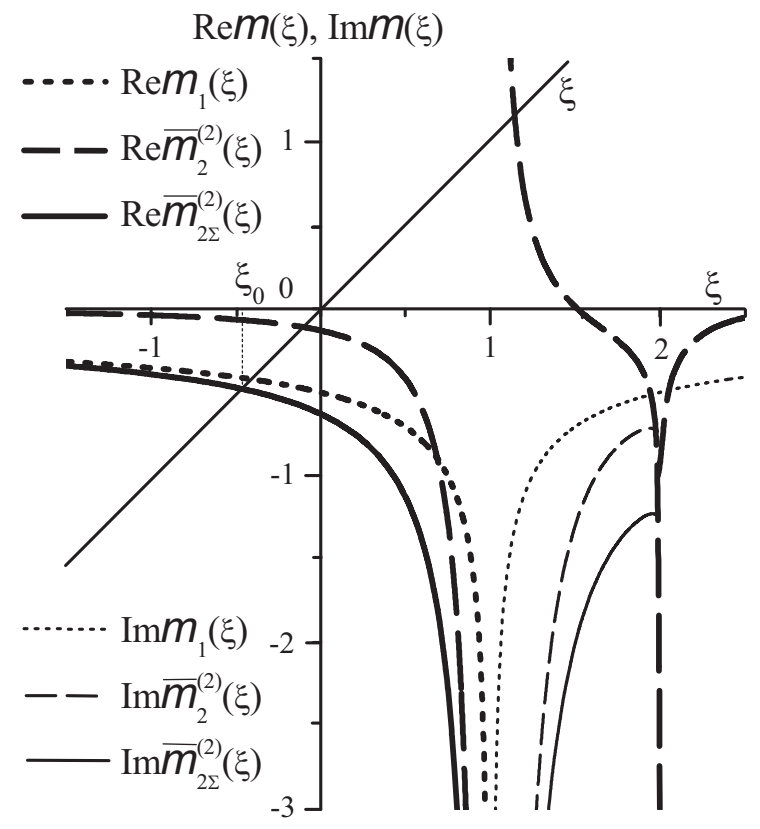

Figure 1. Dependences of $m_{1}, \bar{m}_{2}^{(2)}$ and $\bar{m}_{2 \Sigma}^{2}$ terms on $\xi$ at $\alpha=0.5$.
In figure 1 typical dependences of MO $\bar{m}_{2 \Sigma}^{(2)}(\xi)=m_{1}(\xi)+\bar{m}_{2}^{(2)}(\xi)$ and its terms on $\xi$ calculated at $\alpha=0.5$ are presented. Here, one can see the properties of MO terms and how they influence the formation of renormalized energy of the ground polaron state $\left(\xi_{0}\right)$.

At $\xi \leqslant 1$ the real parts of all terms $\left[\operatorname{Re} \bar{m}_{2 \Sigma}^{(2)}(\xi)\right]$ are negative and regularly decay tending to $-\infty$ at $\xi \rightarrow 1$ from the left. In this region, $\operatorname{Im} \bar{m}_{2 \Sigma}^{(2)}(\xi)=0$, thus, the renormalized energy of polaron ground state is obtained from the expression $\xi-\bar{m}_{2 \Sigma}^{(2)}(\xi)=$ 0 . It is clear from figure 1 that two-phonon $\bar{m}_{2}^{(2)}(\xi)$ being taken into account in addition to the one-phonon $m_{1}(\xi)$, makes $\xi_{0}$ magnitude more precise by increasing its absolute value.

At $\xi \leqslant 1, \operatorname{Re} m_{1}(\xi)=0$ and $\operatorname{Im} m_{1}(\xi)<0$, decaying over the absolute magnitude from $-\infty$ at $\xi=1$ to zero at $\xi \rightarrow \infty$. In the range $1 \leqslant \xi \leqslant 2, \operatorname{Re} \bar{m}_{2}^{(2)}(\xi)$ varies from $\infty$ to $-\infty$ and $\operatorname{Im} \bar{m}_{2}^{(2)}(\xi)$ varies from $-\infty$ to the finite negative value. At $2 \leqslant \xi \operatorname{Im} \bar{m}_{2}^{(2)}(\xi)=0$ and $\operatorname{Re} \bar{m}_{2}^{(2)}(\xi)$ varies from $-\infty$ at $\xi=2$ to zero at $\xi \rightarrow \infty$.

These properties of MO terms determine the features of polaron spectrum manifested through the dependence of spectral density $\rho$ on dimensionless energy $\xi$

$$
\rho(\xi)=-2 \operatorname{Im} g(\xi)=-\frac{2 \operatorname{Im} m(\xi)}{[\xi-\operatorname{Re} m(\xi)]^{2}+[\operatorname{Im} m(\xi)]^{2}} .
$$

In figure 2. the function $\rho(\xi)$ is presented at $\alpha=0.25,0.5,0.75$ calculated within MO $m_{1}(\xi)$ and $\bar{m}_{2 \Sigma}^{(2)}(\xi)$. It is clear that independently of the approximated MO, besides $\delta$-peak corresponding to the renormalized energy of ground polaron state, the asymmetric peak of one-phonon repetition with big width $(\gamma)$ is observed because polaron in this state has a small lifetime. At bigger $\alpha$, the renormalized energy $\left(\xi_{0}\right)$ shifts into the negative region while the position of the maximum of one-phonon repetition $\left(\xi_{1}\right)$ shifts into the region of higher energies with the increasing width $(\gamma)$ of this peak.

Finally, we should note that one- and two-phonon MO, proportional to $\alpha$ and $\alpha^{2}$ respectively, cause the renormalization of polaron ground state energy in such a way that it shifts into the region of smaller energies when $\alpha$ increases, according to the physical considerations. As for the excited state, where polaron is bound with one phonon, it is manifested as asymmetric peak in function $\rho(\xi)$. When $\alpha$ increases, its maximum shifts into the region of higher energies, being incorrect from the physical point of view. In the next section we show that in order to correctly calculate the spectral parameters of the excited states, one should perform a partial summing of infinite ranges of MO diagrams instead of a finite number thereof. 


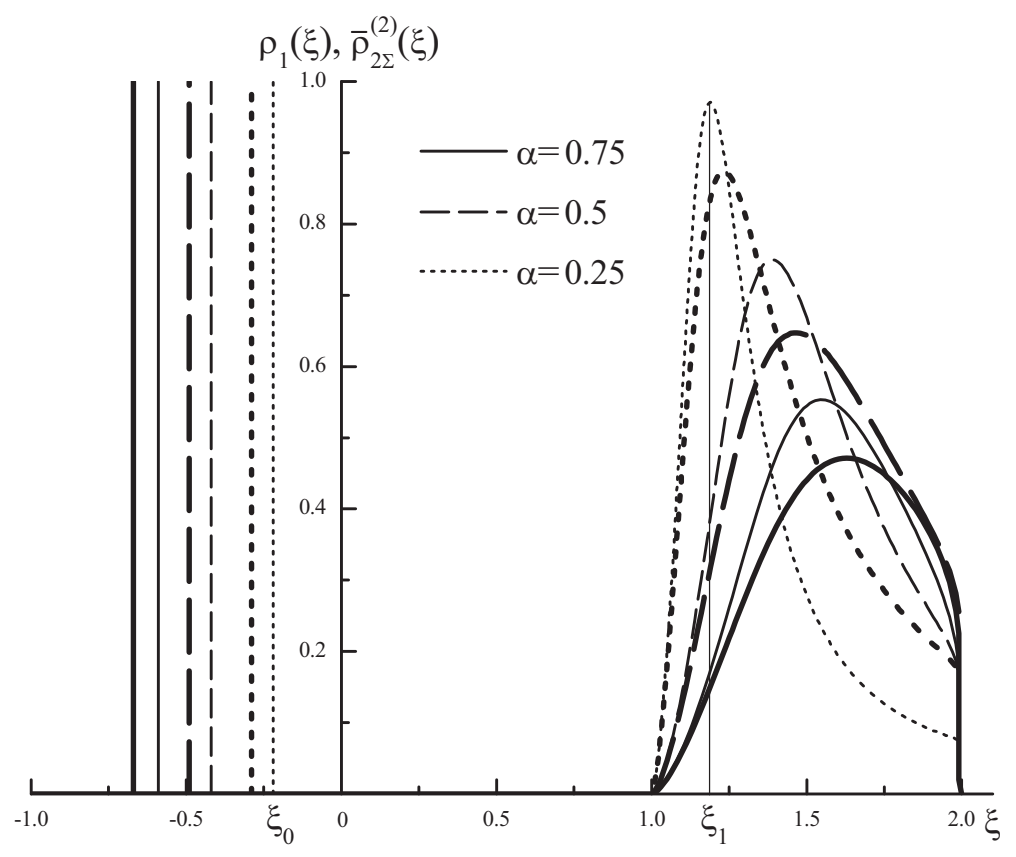

Figure 2. Dependences of spectral density $\rho$ on energy $\xi$ at different values of the coupling constant $\alpha$ within different approximations for MO. $\rho_{1}-$ thin curve, $\bar{\rho}_{2 \Sigma}^{(2)}-$ thick curve.

\section{Ground and first excited polaron states renormalized due to one- and two-phonon processes}

Taking into account the infinite number of diagrams in MO describing one- and two-phonon processes over all powers of the coupling constant but containing no diagram of three- or more phonons, brings us to the physically correct behavior of the renormalized energy both of the ground and the first excited state. We refer to such MO as the two-phonon MO $\left[M_{2}(\vec{k}, \omega)\right]$. In diagrammatic representation it is written, according to the rules of Feynman-Pines diagram technique, as follows:

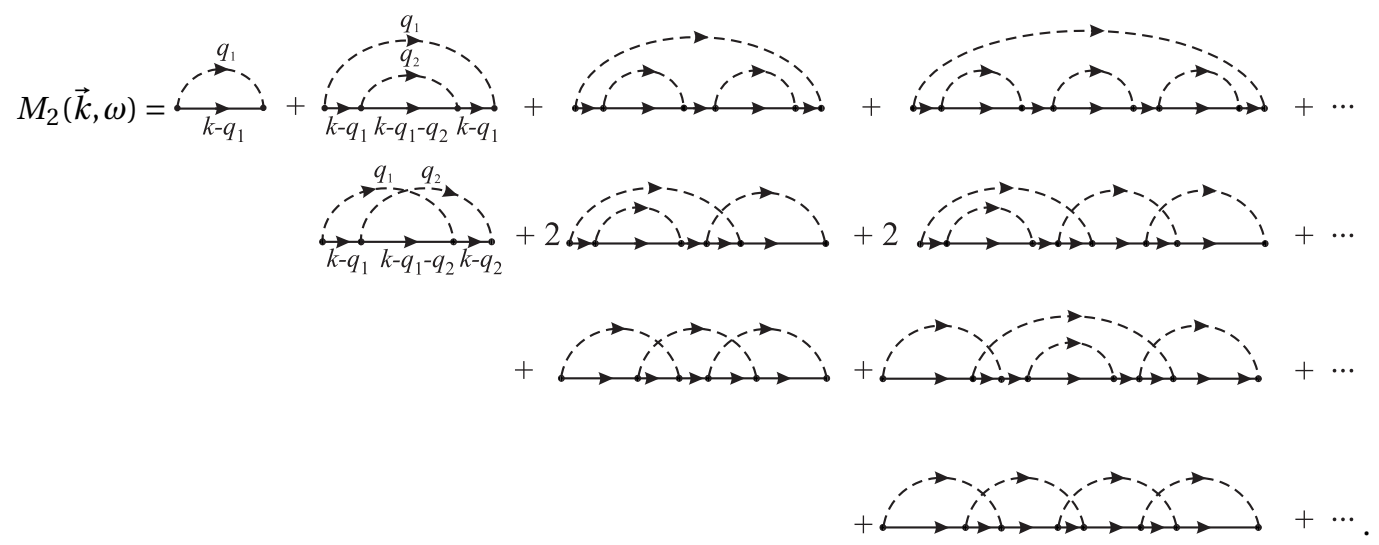

Performing a complete partial summing of this range of diagrams, we obtain

$$
M_{2}(\vec{k}, \omega)=\sum_{\vec{q}_{1}} \frac{\left|\varphi\left(q_{1}\right)\right|^{2}}{\tilde{\varepsilon}_{\vec{k}-\vec{q}_{1}}}+\sum_{\vec{q}_{1} \vec{q}_{2}} \frac{\left|\varphi\left(q_{1}\right)\right|^{2}\left|\varphi\left(q_{2}\right)\right|^{2}}{\tilde{\varepsilon}_{\vec{k}-\vec{q}_{1}} \varepsilon_{\vec{k}-\vec{q}_{1}-\vec{q}_{2}} \tilde{\varepsilon}_{\vec{k}-\vec{q}_{2}}}+\sum_{\vec{q}_{1} \vec{q}_{2} \vec{q}_{3}} \frac{\left|\varphi\left(q_{1}\right)\right|^{2}\left|\varphi\left(q_{2}\right)\right|^{2}\left|\varphi\left(q_{3}\right)\right|^{2}}{\tilde{\varepsilon}_{\vec{k}-\vec{q}_{1}} \varepsilon_{\vec{k}-\vec{q}_{1}-\vec{q}_{2}} \tilde{\varepsilon}_{\vec{k}-\vec{q}_{2}} \varepsilon_{\vec{k}-\vec{q}_{2}-\vec{q}_{3}} \tilde{\varepsilon}_{\vec{k}-\vec{q}_{3}}}+\ldots
$$


where

$$
\tilde{\varepsilon}_{\vec{k}-\vec{q}_{1}}=\varepsilon_{\vec{k}-\vec{q}_{1}}-\sum_{\vec{q}_{2}} \frac{\left|\varphi\left(q_{2}\right)\right|^{2}}{\varepsilon_{\vec{k}-\vec{q}_{1}-\vec{q}_{2}}}=\hbar \omega-E_{\vec{k}-\vec{q}}-\Omega-\sum_{\vec{q}_{2}} \frac{\left|\varphi\left(q_{2}\right)\right|^{2}}{\hbar \omega-E_{\vec{k}-\vec{q}_{1}-\vec{q}_{2}}-2 \Omega}
$$

is the energetic denominator renormalized due to two-phonon processes.

Transiting here from summing to integration, integrating over the angular variables and using the dimensionless parameters (8), we obtain an exact analytical expression for a dimensionless two-phonon MO

$$
m_{2}(\vec{K}, \xi)=\sum_{n} m_{2}^{(n)}(\vec{K}, \xi)
$$

At $K=0$, its terms are as follows:

$$
\begin{aligned}
& m_{2}^{(1)}(\xi)=\frac{2 \alpha}{\pi} \int_{0}^{\infty} \frac{\mathrm{d} Q_{1}}{\xi-Q_{1}^{2}-1+\alpha A\left(\xi, Q_{1}\right)+\mathrm{i} \eta}, \\
& m_{2}^{(2)}(\xi)=\frac{\alpha^{2}}{\pi^{2}} \int_{0}^{\infty} \int_{0}^{\infty} \frac{\mathrm{d} Q_{1}}{Q_{1}} \frac{\mathrm{d} Q_{2}}{Q_{2}} \frac{\ln \left[\frac{2-\xi+\left(Q_{1}-Q_{2}\right)^{2}}{2-\xi+\left(Q_{1}+Q_{2}\right)^{2}}\right]}{\left[\xi-Q_{1}^{2}-1+\alpha A\left(\xi, Q_{1}\right)+\mathrm{i} \eta\right]\left[\xi-Q_{2}^{2}-1+\alpha A\left(\xi, Q_{2}\right)+\mathrm{i} \eta\right]}, \\
& m_{2}^{(n)}(\xi)=4\left(\frac{\alpha}{2 \pi}\right)^{n} \int_{0}^{\infty}(n) \int_{0}^{\infty} \frac{\frac{Q_{2}}{Q_{1}} \prod_{s=2}^{n} Q_{s}^{-2} \ln \left[\frac{2-\xi+\left(Q_{s}-Q_{s-1}\right)^{2}}{2-\xi+\left(Q_{s}+Q_{s-1}\right)^{2}}\right]}{\prod_{s=1}^{n}\left[\xi-Q_{s}^{2}-1+\alpha A\left(\xi, Q_{s}\right)+\mathrm{i} \eta\right]} \mathrm{d} Q_{1} \ldots \mathrm{d} Q_{n}, \quad(n=2,3, \ldots, \infty)
\end{aligned}
$$

where

$$
A(\xi, Q)= \begin{cases}\frac{1}{Q} \arctan \left(\frac{Q}{\sqrt{2-\xi}}\right), & \xi \leqslant 2, \\ \frac{\pi}{Q} \theta(Q-\sqrt{\xi-2})+\frac{\mathrm{i}}{2 Q} \ln \left|\frac{Q+\sqrt{\xi-2}}{Q-\sqrt{\xi-2}}\right|, & \xi \geqslant 2 .\end{cases}
$$

The integrals in formulae (24)-(26) are calculated within numerical methods. They are typical and contain smooth (without peculiarities) functions of real variables [numerator in formula [26] multiplied by generalized functions $\prod_{s=1}^{n}\left[\xi-Q_{s}^{2}-1+\alpha A\left(\xi, Q_{s}\right)+\mathrm{i} \eta\right]^{-1}$. The presence of the latter causes a different integration, depending on the range of $\xi$. There are three specific ranges.

At $\xi \geqslant 2$, all factors

$$
\xi-Q_{s}^{2}-1+\frac{\pi \alpha}{Q_{s}} \theta\left(Q_{s}-\sqrt{\xi-2}\right)+\frac{\mathrm{i} \alpha}{2 Q_{s}} \ln \left|\frac{Q_{s}+\sqrt{\xi-2}}{Q_{s}-\sqrt{\xi-2}}\right|
$$

are complex functions, thus, they are all integrals and, hence, $m_{2}^{(n)}(\xi \geqslant 2)$ is a complex function containing $\operatorname{Re} \boldsymbol{M}_{2}^{(n)}(\xi \geqslant 2)$ and $\operatorname{Im} \boldsymbol{M}_{2}^{(n)}(\xi \geqslant 2)$ parts.

When $\xi \leqslant 2$, the character of the integrals depends on whether there exists any solution of the equation

$$
\xi-Q^{2}-1+\frac{\alpha}{Q} \arctan \frac{Q}{\sqrt{2-\xi}}=0
$$

at fixed $\xi$. At $Q=0$, this equation is rewritten as follows:

$$
\xi+\frac{\alpha}{\sqrt{2-\xi}}=1
$$

which has an exact real solution

$$
\bar{\xi}=2-\frac{4}{3} \begin{cases}\cos ^{2}\left[\frac{1}{3} \arccos \left(\frac{3 \sqrt{3}}{2} \alpha\right),\right. & \alpha \leqslant \frac{2}{3 \sqrt{3}}, \\ \operatorname{ch}^{2}\left[\frac{1}{3} \operatorname{arcch}\left(\frac{3 \sqrt{3}}{2} \alpha\right)\right], & \alpha \geqslant \frac{2}{3 \sqrt{3}}\end{cases}
$$


producing two regions for $\xi \leqslant 2: \xi \leqslant \bar{\xi}$ and $\bar{\xi} \leqslant \xi \leqslant 2$.

At $\xi \leqslant \bar{\xi}$, the equation (28) does not have any solution, thus the functions in respective integrals for $m_{2}^{(n)}(\xi)$ have no poles and, hence, the MO $m_{2}^{(n)}(\xi)$ are real functions.

In the region $\bar{\xi} \leqslant \xi \leqslant 2$, the equation 28 has the solution in the point $Q_{0}$ at fixed $\xi$. Thus, the integrals in $m_{2}^{(n)}(\xi)$ contain this specific point and are calculated using Dirac identity

$$
\int_{0}^{\infty} \frac{\Phi(Q)}{f(Q)+\mathrm{i} \eta} \mathrm{d} Q=\mathscr{P} \int_{0}^{\infty} \frac{\Phi(Q)}{f(Q)} \mathrm{d} Q-\mathrm{i} \frac{\pi}{\left|f^{\prime}(Q)\right|_{Q=Q_{0}}} \int_{0}^{\infty} \Phi(Q) \delta\left(Q-Q_{0}\right) \mathrm{d} Q,
$$

where $\Phi(Q)$ is the regular function and $f\left(Q=Q_{0}\right)=0$.

At $\xi=2$, the equation (28) has an exact real solution

$$
Q_{0}(\xi=2, \alpha)=\frac{2}{\sqrt{3}} \begin{cases}\cos \left[\frac{1}{3} \arccos \left(\frac{3 \sqrt{3} \pi \alpha}{4}\right)\right], & \alpha \leqslant \frac{4}{3 \sqrt{3} \pi}, \\ \operatorname{ch}\left[\frac{1}{3} \operatorname{arcch}\left(\frac{3 \sqrt{3} \pi \alpha}{4}\right)\right], & \alpha \geqslant \frac{4}{3 \sqrt{3} \pi},\end{cases}
$$

thus, the function $Q_{0}(\xi, \alpha)$ smoothly increases from 0 to $Q_{0}(2, \alpha)$ in the range $\bar{\xi} \leqslant \xi \leqslant 2$, figure 3

The presented analysis proves that $m_{2}^{(1)}(\xi)$ is given by an analytical expression

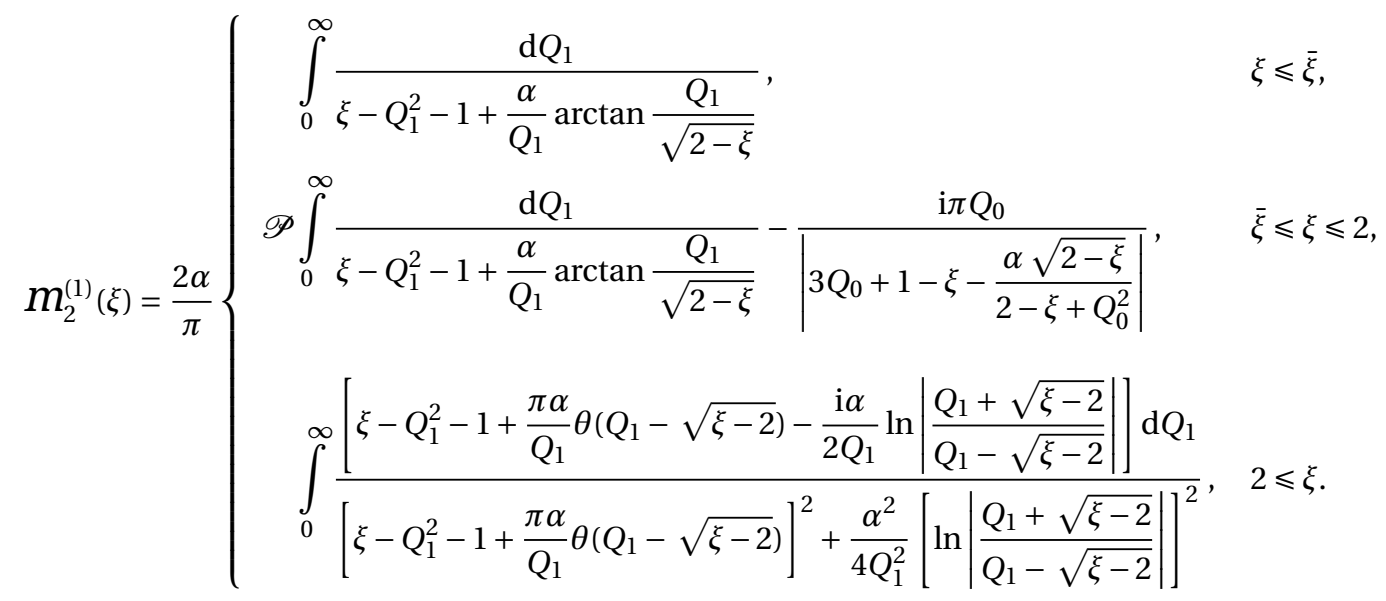

All integrals in $m_{2}^{(n)}(\xi)$ are calculated in a similar way.

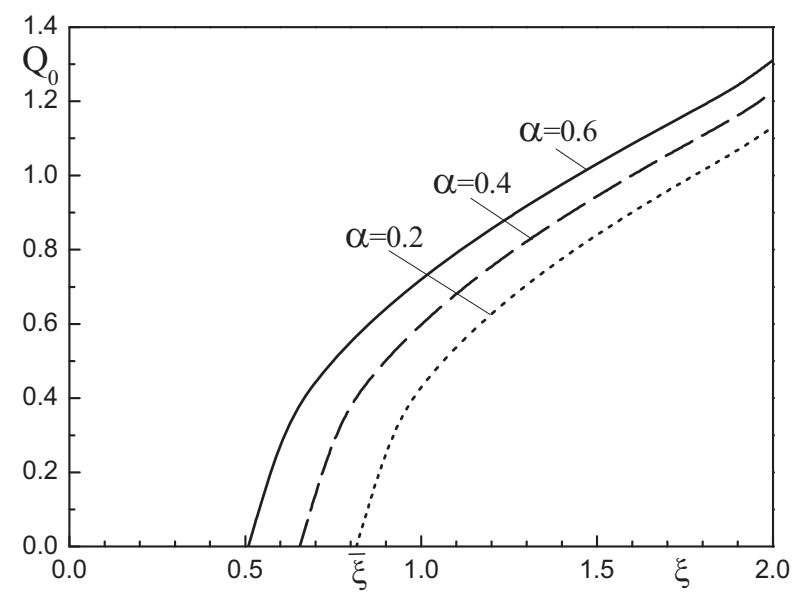

Figure 3. $Q_{0}$ as function of $\xi$ at $\alpha=0.2,0.4,0.6$. 

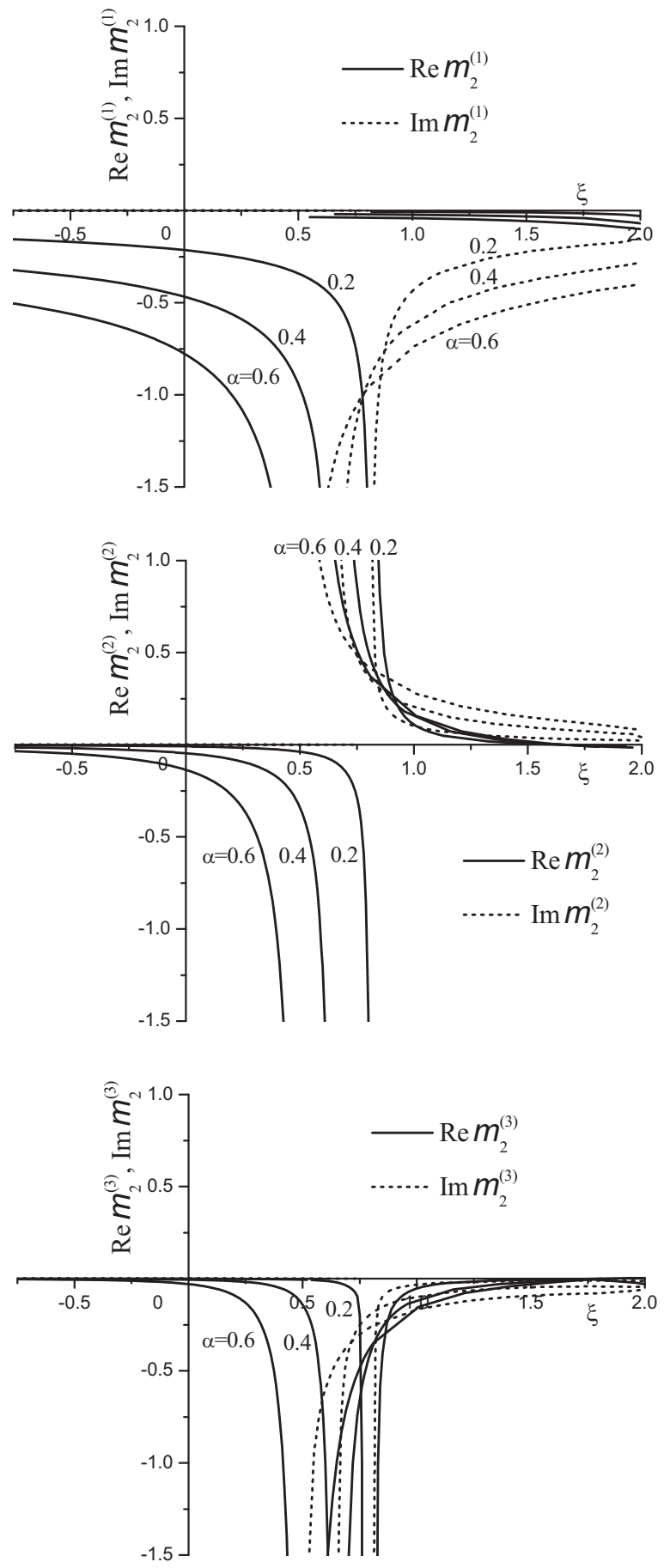

Figure 4. MO $m_{2}^{(n)}$ as a function of $\xi$ at $\alpha=0.2,0.4,0.6$.
The first three terms of MO $m_{2}^{(n=1,2,3)}(\xi)$ at $\alpha=0.2,0.4,0.6$ are shown in figure 4 It is clear that at $\xi \leqslant \bar{\xi}$, $\operatorname{Im}_{2}^{(n)}(\xi)=$ 0 and $\operatorname{Re}_{2}^{(n)}(\xi)<0$ independently of the coupling constant $\alpha$ for all $n$, herein $\lim _{\xi \rightarrow \bar{\xi}} m_{2}^{(n)}(\xi) \rightarrow-\infty$. The absolute magnitude $\left|\operatorname{Re} \boldsymbol{M}_{2}^{(n)}(\xi)\right|$ increases for a bigger coupling constant $\alpha$. In the range $\bar{\xi} \leqslant \xi \leqslant 2$, the real and imaginary parts of $\boldsymbol{m}_{2}^{(1)}(\xi)$ and $m_{2}^{(3)}(\xi)$ are negative while those of $m_{2}^{(2)}(\xi)$ and $m_{2}^{(4)}(\xi)$ are positive.

In figure 5 the functions of real and imaginary parts of MO $m_{2 \Sigma}^{(n=1,2,3)}(\xi)$ (a) and densities of energies $\rho_{2 \Sigma}^{(n=1,2,3)}(\xi)$ (b) are presented at $\xi \leqslant 2$. The region $\xi>2$ is not observed because here for correct results one has to take into account the MO renormalized due to the three-phonon processes. Figure 5 (a) proves that when $\xi \leqslant \bar{\xi}$, $\operatorname{Im} m_{2 \Sigma}^{(n)}(\xi \leqslant$ $\bar{\xi})=0$ and, thus, there is no decay of the renormalized ground state. The real part of MO $\operatorname{Re} m_{2 \Sigma}^{(n)}(\xi \leqslant \bar{\xi})$ is negative and its absolute value increases at bigger $\alpha$. Both features bring us to the fact that the energy density has a $\delta$-like shape $\rho_{2 \Sigma}^{(n)}(\xi)=\delta\left(\xi-\xi_{0 \Sigma}^{(n)}\right)$ with the peak at the renormalized ground state energy.

In the range $\bar{\xi} \leqslant \xi \leqslant 2$, the energy density $\rho_{2 \Sigma}^{(n)}(\xi)$ in all approximations has the shape of asymmetric quasi-Lorentz peak, arising due to the first excited state of polaron, interpreted as a bound state of electron with one phonon. The maximum of $\rho_{2 \Sigma}^{(n)}$ in the energy scale fixes the energy of this bound state and the width $\left(\gamma_{2 \Sigma}^{(n)}\right)$ of the peak at the half of its height defines the decay of this quasistationary state or its lifetime $\left(\tau_{2 \Sigma}^{(n)}=\hbar / \gamma_{2 \Sigma}^{(n)}\right)$.

We should note that in spectroscopy, according to the physical characteristics of renormalized ground and the first excited states, the respective peaks of the function $\rho_{2}(\xi)$ are referred to as phononless and onephonon repetitions. The function $\rho_{2 \Sigma}^{(n)}(\xi)$ and its spectral parameters $\left(\xi_{0 \Sigma}^{(n)}, \xi_{1 \Sigma}^{(n)}, \gamma_{1 \Sigma}^{(n)}\right)$ are shown in figure 5 (b) for different $\alpha$ and MO approximation $\left(\boldsymbol{M}_{2 \Sigma}^{(n)}\right)$.

According to the physical considerations and the behavior of optical conductivity at small $\alpha$ revealed in paper [14], the energies of both states shift into the low-energy region when the coupling constant increases. Herein, the ground state is a stationary one (not decaying) and the decay of the excited quasi-stationary state increases.

The spectral parameters also depend on the approximation of MO $m_{2 \Sigma}^{(n)}$. Figure 5 (b) and table 1 prove 

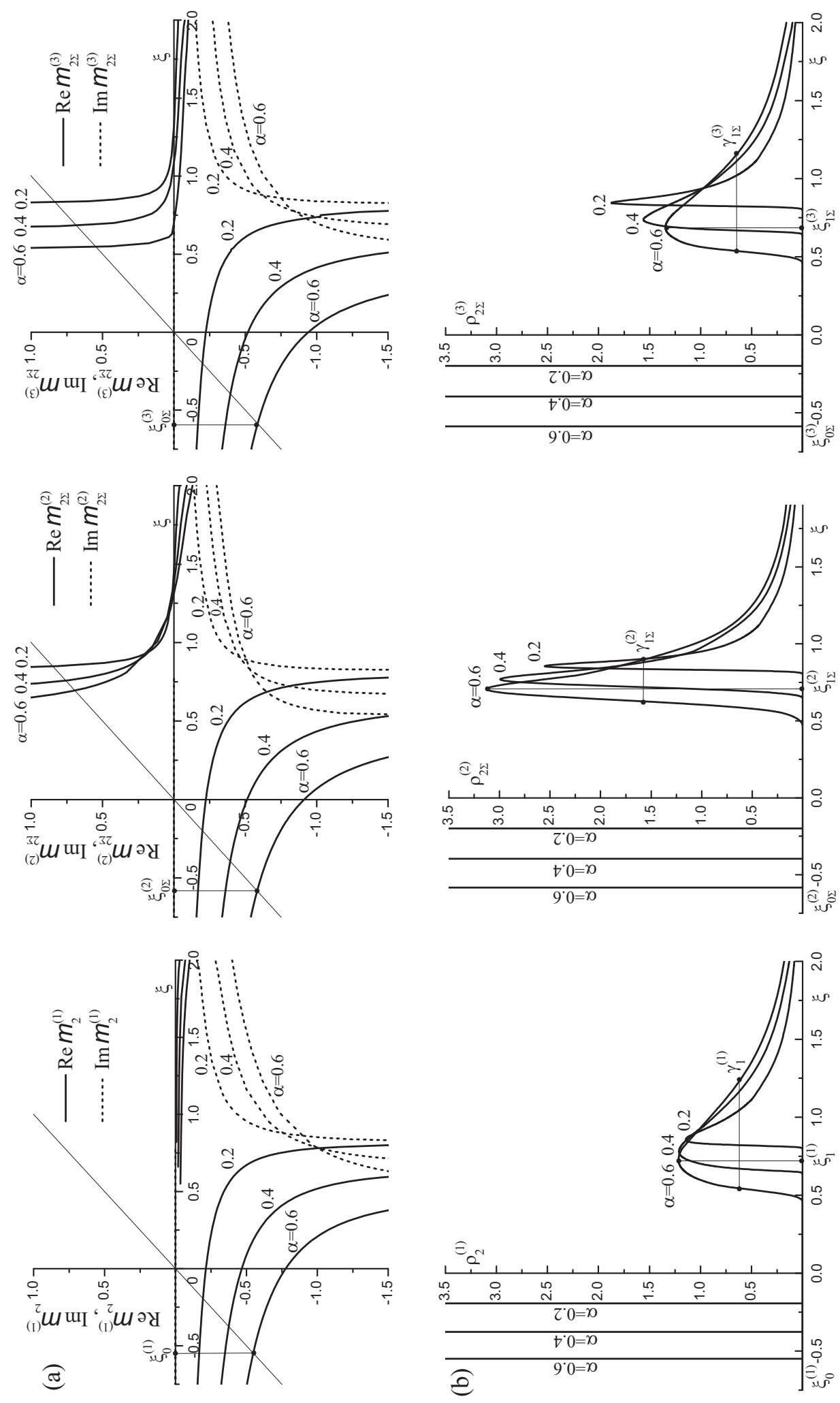

Figure 5. MO $m_{2 \Sigma}^{(n)}$ (a) and energy density $\rho_{2 \Sigma}^{(n)}$ (b) as functions of $\xi$ at $n=1,2,3$ and $\alpha=0.2,0.4,0.6$. 
Table 1. Dependences of the spectral parameters in the first three orders on the magnitude of $\alpha$.

\begin{tabular}{|c||c|c|c|}
\hline$\alpha$ & 0.2 & 0.4 & 0.6 \\
\hline \hline$\xi_{0}^{(1)}$ & -0.194 & -0.376 & -0.548 \\
\hline$\xi_{1}^{(1)}$ & 0.856 & 0.780 & 0.728 \\
\hline$\xi_{1}^{(1)}-\xi_{0}^{(1)}$ & 1.050 & 1.154 & 1.276 \\
\hline$\gamma_{1}^{(1)}$ & 0.247 & 0.494 & 0.694 \\
\hline \hline$\xi_{0 \Sigma}^{(2)}$ & -0.199 & -0.395 & -0.583 \\
\hline$\xi_{1 \Sigma}^{(2)}$ & 0.854 & 0.767 & 0.705 \\
\hline$\xi_{1 \Sigma}^{(2)}-\xi_{0 \Sigma}^{(2)}$ & 1.053 & 1.162 & 1.288 \\
\hline$\gamma_{1 \Sigma}^{(2)}$ & 0.077 & 0.172 & 0.273 \\
\hline \hline$\xi_{0 \Sigma}^{(3)}$ & -0.20 & -0.396 & -0.585 \\
\hline$\xi_{1 \Sigma}^{(3)}$ & 0.84 & 0.74 & 0.69 \\
\hline$\xi_{1 \Sigma}^{(3)}-\xi_{0 \Sigma}^{(3)}$ & 1.04 & 1.136 & 1.275 \\
\hline$\gamma_{1 \Sigma}^{(3)}$ & 0.118 & 0.374 & 0.594 \\
\hline
\end{tabular}

that the energies of the ground and the excited states $\left(\xi_{0 \Sigma}^{(n)}, \xi_{1 \Sigma}^{(n)}\right)$ for bigger $n$ are uniformly defined more precisely, while the decay $\gamma_{2 \Sigma}^{(n)}$ is defined varying between minimal values at odd $n$ and maximal values at even ones. From the table 1 it is clear that at small $\alpha$, the difference between the energies of the ground and excited states correlates with the magnitude of the phonon energy. At a bigger $\alpha$ it increases, which is probably not a physical property but the result of insufficiency of two-phonon approximation in MO used for the calculation of renormalized energies.

\section{Conclusions}

Using the Feynman-Pines diagram technique, the exact analytical calculation of MO for the Fourier image of polaron Green's function is performed in the first and the second order over the electron-phonon coupling constant. It is shown that though such an approximation makes the renormalized energy of polaron ground state more precise, but even at a weak coupling $(\alpha<1)$ the energy and decay of the first excited state is evaluated very roughly. Firstly, the peak of the energy density of the first phonon repetition in the energy scale is located higher than the energy $E+\Omega$, however, according to the physical considerations it should be lower because it is produced by the interaction between electron and virtual phonon ( $T=0 \mathrm{~K})$. Secondly, the increasing coupling constant causes the shift of the peak of one-phonon repetition into the high-energy region, which is not correct either.

A partial summing of all the infinite range of MO diagrams that do not contain three- and more phonon energies is performed. The two-phonon MO and the energy density are calculated and their properties are analyzed for the renormalized ground and first excited polaron states. It is shown, for the first time, that according to the physical considerations, the stronger electron-phonon interaction, i.e., an increasing coupling constant, shifts the energy of the ground and first excited polaron states into the low-energy region. Herein, the ground state stays stationary and the decay of the excited state increases. At small $\alpha$, the difference between the energies of the ground and the excited polaron states correlates with the magnitude of the phonon energy.

The developed approach of partial summing of diagrams containing three-, four- and $n$-phonon processes in polaron MO principally makes it possible to obtain more accurate renormalized energies and decays both of the ground state and multi-phonon repetitions. However, an increasing number of repetitions and the exactness of their spectral parameters brings us to complicated analytical and numeric calculations. 


\title{
References
}

1. Landau L.D., Phys. Z. Sowjetunion, 1933, 3, 664.

2. Landau L.D., Pekar S.I., Zh. Eksp. Teor. Fiz., 1948, 18, 419 (in Russian).

3. Bogolubov N.N., Tyablikov S.V., Zh. Eksp. Teor. Fiz., 1949, 19, 256 (in Russian).

4. Lee T.D., Low F.E., Pines D., Phys. Rev., 1953, 90, 297; doi 10.1103/PhysRev.90.297

5. Fröhlich H., Adv. Phys., 1954, 3, 325; doi $10.1080 / 00018735400101213$

6. Feynman R.P., Phys. Rev., 1955, 97, 660; doi 10.1103/PhysRev.97.660

7. Whitfield D., Puff R., Phys. Rev. A, 1965, 139, 338; doi 10.1103/PhysRev.139.A338.

8. Haeringer W.V., Phys. Rev. A, 1965, 137, 1902; doi 10.1103/PhysRev.137.A1902

9. Devreese J.T., Evrard R., Phys. Lett., 1964, 11, 278; doi 10.1016/0031-9163(64)90324-5.

10. Kartheuser E., Evrard R., Devreese J., Phys. Rev. Lett., 1969, 22, 94; doi 10.1103/PhysRevLett.22.94

11. Devreese J., De Sitter J., Goovaerts M., Solid State Commun., 1971, 9, 1383; doi 10.1016/0038-1098(71)90400-5

12. Devreese J., De Sitter J., Goovaerts M., Phys. Rev. B, 1972, 5, 2367; doi 10.1103/PhysRevB.5.2367

13. Peeters F.M., Devreese J.T., Phys. Rev. B, 1983, 28, 6051; doi 10.1103/PhysRevB.28.6051

14. Mishchenko A.S., Prokof'ev N.V., Sakamoto A., Svistunov B.V., Phys. Rev. B, 2000, 62, 6317; doi 10.1103/PhysRevB.62.6317

15. Mishchenko A.S., Nagaosa N., Prokof'ev N.V., Sakamoto A., Svistunov B.V., Phys. Rev. Lett., 2003, 91, 236401; doi $10.1103 /$ PhysRevLett.91.236401.

16. De Filippis G., Cataudella V., Mishchenko A.S., Perroni C.A., Devreese J.T., Phys. Rev. Lett., 2006, 96, 136405; doi 10.1103/PhysRevLett.96.136405

17. Mishchenko A.S., Phys.-Usp., 2005, 48, 887; doi 10.1070/PU2005v048n09ABEH002632

18. Devreese J.T., J. Phys.: Condens. Matter, 2007, 19, 255201; doi 10.1088/0953-8984/19/25/255201

19. Devreese J.T., Alexandrov A.S., Rep. Prog. Phys., 2009, 72, 1; doi $10.1088 / 0034-4885 / 72 / 6 / 066501$

20. Mishchenko A.S., Phys.-Usp., 2009, 52, 1193; doi 10.3367/UFNe.0179.200912b.1259.

21. De Filippis G., Cataudella V., Mishchenko A.S., Nagaosa N., Phys. Rev. B, 2012, 85, 094302; doi $10.1103 /$ PhysRevB.85.094302

22. Tkach M.V., Quasiparticles in Nanoheterosystems. Quantum Dots and Wires, Chernivtsi University Press, Chernivtsi, 2003.

23. Pokatilov E.P., Klimin S.N., Fomin V.M., Devreese J.T., Wise F.W., Phys. Rev. B, 2002, 65, 075316; doi 10.1103/PhysRevB.65.075316

24. Gladilin V.N., Klimin S.N., Fomin V.M., Devreese J.T., Phys. Rev. B, 2004, 69, 155325; doi 10.1103/PhysRevB.69.155325

25. Bennett C.R., Tanatar B., Phys. Rev. B, 1997, 55, 7165; doi 10.1103/PhysRevB.55.7165.

26. Tkach M.V., Seti Ju.O., Grynyshyn Y.B., Voitsekhivska O.M., Condens. Matter Phys., 2014, 17, 23704; doi 10.5488/CMP.17.23704

27. Tkach M.V., Seti Ju.O., Grynyshyn Ju.B., Ukr. J. Phys., 2014, 59, 1191.

28. Abrikosov A.A., Gorkov L.P., Dzyaloshinski I.E., Methods of Quantum Field Theory in Statistical Physics., Dover, New York, 2012.

\section{Перенормована енергія основного і першого збудженого стану полярона Феліха зі слабким зв'язком}

\author{
М.В. Ткач, Ю.О. Сеті, О.М. Войцехівська, О.Ю. Питюк \\ Чернівецький національний університет ім. Ю. Федьковича, \\ вул. Коцюбинського, 2, 58012 Чернівці, Україна
}

\begin{abstract}
Методом діаграмної техніки Фейнмана-Пайнса виконано парціальне підсумовування безмежного ряду діаграм двофононного масового оператора полярона, що описується гамільтоніаном Фреліха. Перенормовані спектральні параметри основного та першого збудженого поляронного стану (фононного повторення) коректно розраховані для електрон-фононної системи зі слабким зв'язком при $T=0$ К. Показано, що сильніша електрон-фононна взаємодія зміщує енергії обох станів у низькоенергетичну область спектра. Основний стан залишається стаціонарним, а загасання збудженого - зростає при збільшенні константи зв'язку.
\end{abstract}

Ключові слова: полярон, фонон, електрон-фононна взаємодія, функція Гріна, масовий оператор 